\title{
A New Metric for Indian Monsoon Rainfall Extremes*
}

\author{
TACKSEUNG Jun AND LALiTH MunAsinghe \\ Barnard College, Columbia University, New York, New York \\ DAVID H. RIND \\ NASA Goddard Institute for Space Studies, New York, New York
}

(Manuscript received 10 December 2013, in final form 22 December 2014)

\begin{abstract}
Extreme monsoon rainfall in India has disastrous consequences, including significant socioeconomic impacts. However, little is known about the overall trends and climate factors associated with extreme rainfall because rainfall greatly varies across India and because few appropriate methods are available to measure extreme rainfall in the context of such heterogeneity. To provide a comprehensive assessment of extreme monsoon rainfall, the authors developed a metric using record rainfall data to measure the changes in the likelihood of extreme high and extreme low rainfall over time; this metric is independent of the characteristics of the underlying rainfall distributions. Hence, the metric is ideally suited to aggregate extreme rainfall information across heterogeneous regions covering India. The authors found that from 1930 to 2013, the likelihood of extreme high and extreme low rainfall increases 2-fold and 4-fold, respectively. These overall trend increases are driven by anomalous increases, particularly in the early 2000s; the likelihood of extreme high and extreme low rainfall increases 5-fold and 18-fold in 2005 and 2002, respectively. These findings imply a broadening of the underlying monsoon rainfall distribution over the past century. The authors also show that the time patterns of the likelihood of extreme rainfall in recent decades are correlated with El Niño-Southern Oscillation, especially when it is in the same phase with the Pacific decadal oscillation and Indian Ocean dipole.
\end{abstract}

\section{Introduction}

Extreme high and extreme low rainfall, particularly in India during the monsoon season (June-September), can result in major catastrophes, such as soil erosion (MartinezCasasnovas et al. 2002), flash floods (Guhathakurta et al. 2011), flood-damaged crops (Gadgil and Kurma 2006), and droughts (Kumar et al. 2013). Therefore, understanding the changes in extreme rainfall events across India is important for developing better disaster management and mitigation policies.

Several studies have examined extreme rainfall events in India (Goswami et al. 2006; Ajayamohan and Rao 2008; Rajeevan et al. 2008; Ghosh et al. 2009, 2012;

\footnotetext{
* Supplemental information related to this paper is available at the Journals Online website: http://dx.doi.org/10.1175/JCLI-D-13-00764.s1.

Corresponding author address: T. Jun, Dept. of Economics, Barnard College, Columbia University, 3009 Broadway, New York, NY 10027.

E-mail: tj32gm@gmail.com
}

Krishnamurthy et al. 2009). The methodology used in these studies is based on the frequency of rainfall exceeding a threshold value. We refer to this framework as the fixed threshold method. Although this approach has intuitive appeal, the fixed threshold method has inherent limitations that prevent a comprehensive assessment of extreme rainfall across India. First, the choice of a threshold value is ad hoc because it depends on the empirics of the underlying rainfall distribution. Second, the study of changes at the very extremes of a distribution is hindered by the fact that, at high threshold values, the observations that exceed the threshold may result in small sample size issues. Third, the most severe limitation, in our view, is that this approach is applicable only to regions that have homogeneous rainfall. Hence, this fixed threshold method is not well suited to synthesize changes in extreme rainfall throughout India, where vastly different regional rainfall distributions occur. Note that the regional heterogeneity in Indian monsoon rainfall is the primary obstacle in assessing the overall trends in the monsoon rainfall (Ghosh et al. 2009, 2012; Krishnamurthy et al. 2009), and this heterogeneity has 
become more pronounced in recent years (Ghosh et al. 2012). However, as noted in Goswami et al. (2006), using a fixed threshold to define extreme rainfall events is justified only if the area it covers is reasonably homogeneous. Otherwise, extreme rainfall in one region may be considered "normal" in another region. Therefore, this approach inevitably restricts the scope of the analysis to central India, where the rainfall distribution is assumed to be fairly uniform. As a consequence, the behavior of extreme rainfall throughout India is not well understood.

In principle, this fixed threshold method can be adapted to analyze heterogeneous rainfall regions by defining different (and possibly time-varying) thresholds to accommodate the differences in rainfall distributions across regions. By assessing the changes in the aggregate count of rainfall events that exceed these various threshold values, an overall measure of changes in extremes can be obtained. However, implementing this concept is not feasible given the amount of information that would be needed on the specifics of rainfall distributions in each region to select comparable thresholds.

The method we propose is essentially a generalization of the concept that ascribes a rainfall distribution to each region. The major difference is that our method does not require any information on the specifics of the underlying distributions to aggregate extreme outcomes across diverse regions. More specifically, our metric is exclusively based on record rainfall indicators that are independent of the underlying distribution. As a consequence, this approach is ideally suited to study extreme rainfall behavior across heterogeneous regions, such as India.

Using the empirical frequency of record observations to infer changes in the underlying distribution is certainly not new (Yang 1975), and this concept has been applied in climate science (Benestad 2003; Meehl et al. 2009). The aim of these applications is to exploit the difference between the predicted (assuming stationarity) and the observed likelihood of a record to determine changes in the underlying distributions. However, these analyses have been solely limited to the characterization of record events. In our study, we advance this methodological framework, whereby we use record information to infer the likelihood of extreme events.

Our method also complements extreme value theory (EVT), which is a widely known approach for characterizing changes in the tails of distributions. EVT has been applied extensively to measure climate extremes (Zwiers and Kharin 1998; Min et al. 2011). However, the estimation procedures based on EVT are also based on events that exceed a preselected threshold. Therefore, in the context of heterogeneous rainfall distributions, as is the case of India, the same issues of "standardizing" thresholds across regions arise (Min et al. 2011).

A further contribution of this study is an analysis of extreme low rainfall. Previous research has largely neglected Indian monsoon droughts, even though droughts can be as economically important as extreme high rainfall, particularly because of their adverse effects on agricultural productivity (Kumar et al. 2013). In this study, we provide a detailed analysis of the changes in the likelihood of extreme low rainfall across India.

Given that our method allows us to compute an aggregate measure of the likelihood of an extreme high and an extreme low rainfall for the entirety of India, we assess the statistical correlation between these likelihoods and various climate factors that are known to be related to monsoon rainfall, such as El Niño and the Indian Ocean dipole. Although numerous studies examined the correlation using mean monsoon rainfall, no studies have associated the climate factors with extreme monsoon rainfall over India. An exception is the study by Ajayamohan and Rao (2008), in which the relationship between extreme daily rainfall in central India and the Indian Ocean dipole mode was examined. Hence, we address this gap in the literature by analyzing the statistical correlation between related climate factors and the likelihood of extreme rainfall.

The remainder of the paper is organized as follows. In section 2, we present the data description, the basic theory, the derivation of our metric, and the estimation framework. In the theory subsection, we illustrate the construction of our metric with a simple example to clarify the logic behind our approach. Section 3 presents our main results on the changes in the likelihood of extreme high and extreme low rainfall across India. Section 4 discusses the results from our correlation analyses between the likelihood of extreme rainfall and climate factors related to monsoon rainfall. Section 5 concludes with a brief summary and a further discussion.

\section{Data and methods}

\section{a. Data}

We use gridded monsoon [June-September (JJAS)] rainfall data with a $0.25^{\circ} \times 0.25^{\circ}$ resolution for $1901-$ 2013 based on rainfall gauge stations in India. The original station data and construction of the grid points are discussed in Pai et al. (2014). The gridded data points are based on daily rainfall data from a total of 6995 rainfall stations over India; the data are interpolated by an inverse distance-weighted scheme to create grid points. As a result, our panel consists of 4964 evenly spaced grids spanning the entirety of India. An average 
of 2600 stations per year is used to create the daily grid points; note that the number of stations per year is not constant. Because the original data are recorded daily, we sum them over a month and then construct a monthly time series [section $2 \mathrm{~b}(6)$ ].

We also attempt to measure the statistical correlation between the likelihood of extreme rainfall and climate factors that are potentially associated with monsoon rainfall. Among the oceanic factors, we use El NiñoSouthern Oscillation (ENSO), the Indian Ocean dipole (IOD), Indian Ocean surface temperature (IOSST), and the Pacific decadal oscillation (PDO). The following is the list of data sources:

- ENSO: ENSO is represented by the mean Niño-3 index from June to September during 1901-2013 and is based on the HadISST1 dataset (Rayner et al. 2003).

- IOD: The IOD during 1901-2013 is the anomalous SST gradient between the western equatorial Indian Ocean $\left(10^{\circ} \mathrm{S}-10^{\circ} \mathrm{N}, 50^{\circ}-70^{\circ} \mathrm{E}\right)$ and the southeastern equatorial Indian Ocean $\left(10^{\circ} \mathrm{S}-0^{\circ}, 90^{\circ}-110^{\circ} \mathrm{E}\right)$ and is based on the Hadley Centre Sea Surface Temperature dataset, version 3.1 (HadSST 3.1.0.0; Kennedy et al. 2011a,b).

- IOSST: The area-weighted mean sea surface temperature (SST) of the tropical Indian Ocean during19012013 is computed for the region of $24^{\circ} \mathrm{S}-24^{\circ} \mathrm{N}, 40^{\circ}-120^{\circ} \mathrm{E}$ and is based on HadSST 3.1.0.0 (Kennedy et al. 2011a,b).

- PDO: The PDO index during 1901-2013 is derived from the leading principal component of monthly SST anomalies in the North Pacific Ocean (poleward of $20^{\circ} \mathrm{N}$ ) (Mantua et al. 1997). The PDO time series is available from the Joint Institute for the Study of the Atmosphere and Ocean at the University of Washington.

Note that the dominant variability in the Indian Ocean is from ENSO, which accounts for $37 \%$ of the Indian Ocean variability (Saji et al. 1999). Therefore, the variability in the IOSST and in IOD are partly contributed by ENSO. We remove the ENSO signal embedded in the IOSST and IOD by using regression techniques from Clark et al. (2000).

Among the atmospheric factors, we consider snow cover extent (SCE) over Eurasia, the North Atlantic Oscillation (NAO), and the Northern Hemisphere surface air temperature ( $\mathrm{NH}$ temperature). The gridded SCE data are from the Northern Hemisphere SCE version 1 by the Rutgers Global Snow Laboratory. The SCE data cover the period from October 1966 to June 2014. In addition to snow cover, the NAO in the winter is used to represent atmospheric variability in the premonsoon season. The NAO index from 1901 to 2013 is the time series of the leading empirical orthogonal function (EOF) of sea level pressure anomalies over the Atlantic region defined by the following coordinates: $20^{\circ}-80^{\circ} \mathrm{N}, 90^{\circ} \mathrm{W}-40^{\circ} \mathrm{E}$ (Hurrell 1995). The surface air temperature in the Northern Hemisphere is based on the NASA GISS surface temperature dataset (Hansen et al. 2010).

\section{b. Methods}

\section{1) Simple illustration}

Before we fully develop our metric, we present a simple example to illustrate the method. We focus on exploiting the record rainfall information to infer changes in the tails of the underlying distribution, without relying on information about the distribution.

Statistically, the monsoon rainfall system is governed by some underlying distribution, and each observed rainfall is a random draw (or sample) from this distribution. We define a rainfall observation from a station as a record high (low) if it is highest (lowest) among all previous observations at the same station (and the same month). Suppose that there are a large number of stations reporting rainfall in each time period. The basics of record theory (Rényi 1962) imply that, if the underlying rainfall distribution is time invariant [i.e., independent and identically distributed (i.i.d.)], then the likelihood of a record high in a given time period is simply the inverse of the elapsed time periods over which the records are kept. More simply, the likelihood of a record high in the second period is $1 / 2$, because two draws (over two periods) are sampled from the same distribution; thus, for every two draws, each draw is equally likely to be higher than the other draw. By the same argument, the likelihood of observing a record high in the third period is $1 / 3$.

The actual pattern of rainfall records may not match this pattern predicted by a stationary distribution. The discrepancy between the predicted (assuming stationarity) and the observed frequency of a record implies a change in the underlying distribution. The technical challenge is how to measure a shift in the underlying distribution that would be consistent with this discrepancy. Our solution is to estimate an implied sampling rate, what we call record equivalent draws (RED), from the same distribution that would match the empirical frequency of the records. Hypothetically, we pretend to sample more (or less) random draws from the same original distribution until the predicted record high rate equals the higher (or lower) observed record high rate.

For example, under the stationary distribution, it is expected that one-half of the stations will report a record high in the second period. In contrast, suppose $75 \%$ of the stations actually report a record high rainfall. To explain this difference between the predicted and the observed frequency of the record high, consider the following hypothetical scenario: the observed rainfall in 
the first period was from a single draw from the rainfall distribution, whereas the observed rainfall at each station in the second period was the maximum of three independent draws from an identical rainfall distribution. Because each of the four draws (one from the first period and three from the second period) is equally probable to be the highest, the likelihood of observing a record high in the second period is $3 / 4$.

In the third period, suppose $50 \%$ of the stations report record highs. Given that the total draws in the first two periods add up to four, an equal number of hypothetical draws in the third period are required to predict that $50 \%$ of the stations will report a record high in the third period. Therefore, the estimated sequence of RED for the first three periods is 1,3 , and 4 . By proceeding similarly for the later time periods, the number of hypothetical draws for each year can be computed by exploiting the difference between the predicted and the observed likelihood of the record highs. The same construction applies to the number of draws based on record lows. This is the number we refer to as RED. We show that RED is a proxy for the likelihood of extreme events [section $2 b(5)]$.

Note that the above procedure presupposes that the data are balanced (i.e., all the time series data cover the same time period without any missing information). Therefore, in the case of balanced data, we only need the empirical frequency of records in each time period to compute RED. Hence, the implementation of our method is both intuitive and computationally similar to the fixed threshold approach. If the underlying data are unbalanced, then the computation of RED is more complicated because we need to use maximum likelihood techniques. We detail this procedure in section $2 \mathrm{~b}(4)$.

\section{2) RECORD THEORY}

Before we present our multiple-draw framework, we start with the classic single-draw framework that implies a stationary rainfall distribution. Specifically, let $k$ denote a composite index for month $i$ and grid $j$. For each $k$, a single rainfall observation is randomly drawn from a continuous and strictly increasing rainfall distribution function $F^{k}(\cdot)$ in each year $t=1,2, \ldots, T$. Let $\left\{x_{t}^{k}\right\}$ denote a sequence of such rainfall draws that are independent random variables for each $k$. We call a rainfall observation a "record high" at time $t$ if it is the highest rainfall among all previous $t-1$ number of observations. We similarly define a record low using this method. The rainfall at time 1 is trivially a high or low record. Under our assumptions, it is inconsequential whether we refer to record highs or record lows (Chandler 1952); hence, we only refer to record high rainfalls in the following discussion (i.e., simply "record"). To formally define a record, we first denote $V_{r}^{k}$ as the $r$ th time for the record high in $k$ and define it inductively as follows:

$$
V_{r}^{k}=\min \left(t \mid t>V_{r-1}^{k}, x_{t}^{k}>x_{V_{r-1}^{k}}^{k}\right), \quad \text { where } \quad V_{1}^{k}=1 .
$$

The variable $e_{t}^{k}$ is the record high indicator in year $t$ for $k$, and it is defined as follows: $e_{t}^{k}=1$ if for some $r, V_{r}^{k}=t$; otherwise, $e_{t}^{k}=0$.

The key result (Rényi 1962) is as follows: under the i.i.d. assumption of the rainfall distribution, the record indicators $e_{2}^{k}, e_{3}^{k}, \ldots$ are independent Bernoulli variables with the following probability:

$$
P\left(e_{t}^{k}=1\right)=1 / t .
$$

A new record is set at time $t$ if and only if the observation at time $t$ is the maximum of the first $t$ realizations. Because each rainfall observation is drawn from the same stationary distribution, each realization has the identical probability of being the maximum. This probability is independent of the underlying rainfall distribution, and it does not depend on the specifics of the distribution.

This nonparametric nature of record processes is key for synthesizing record monsoon rainfall observations from different geographic regions. Hence, one station may be located in the hot and dry zone in central India, and a second station may be in a warm and humid zone along the coast. Therefore, we drop the index $k$ from the definitions of the distribution and record indicators and simply denote them as $F(\cdot)$ and $e_{t}$.

\section{3) Construction of RED}

Here, we introduce time-varying multiple draws to drop the i.i.d. assumption and incorporate changes in the underlying distribution. Let $F_{t}(\cdot)$ denote the rainfall distribution at time $t$ such that it is now time dependent. Suppose that for $s>t, F_{s}(\cdot)$ represents a "wetter" distribution than $F_{t}(\cdot)$. We express this relationship as follows:

$$
F_{s}(\cdot)=F_{t}^{\gamma(t, s)}(\cdot) .
$$

By induction, this becomes

$$
F_{s}(\cdot)=F_{1}^{\gamma(1, s)}(\cdot),
$$

where $\gamma(1, s) \geq 1$. Equation (2) implies that the distribution $F_{s}(\cdot)$ is generated by taking the maximum of $\gamma(1, s)$ draws from the initial (or base) distribution $F_{1}(\cdot)$. Thus, the likelihood of observing rainfall smaller than some fixed value is lower in year $s$ than in the initial year. The number of draws at any time is measured relative to the number of draws in the initial year. 
Because draws in any given time period are assumed to arrive from the same base distribution, Rényi's (1962) result in Eq. (1) holds in this multidraw context as follows: the record indicators $e_{2}, e_{3}, \ldots$ are independent Bernoulli variables with the probability

$$
P\left(e_{t}=1\right)=\gamma(t-1, t) / \sum_{\tau>1}^{t} \gamma(\tau-1, \tau) .
$$

As a consequence, the number of draws in any time period is independent of the rainfall distribution. In the next section, we derive an empirical counterpart of Eq. (3) to estimate the number of draws based on the record rainfall data.

\section{4) Estimation OF RED}

Let $c_{t}$ (RED) be the empirical counterpart of $\gamma$ in year $t$, defined as the area-weighted (by the cosine of the latitude) number of draws over the 4-month time series (representing the 4 monsoon months per year) and grids in year $t$. The total number of draws until time $t$ is defined as $\lambda_{t}=\sum_{\tau=1}^{t} c_{\tau}$. Then, the probability that a record will be set in year $t$ is

$$
P\left(e_{t}=1\right)=c_{t} / \lambda_{t},
$$

which is the empirical counterpart of Eq. (3). If $c_{t}$ is constant, then both the probability in Eqs. (3) and (4) simplify to $1 / t$, which is the classical case with a constant draw rate.

Our empirical strategy is to estimate the $\left\{c_{t}\right\}$ sequence using the maximum likelihood estimation. Let $n_{t}$ and $b_{t}$ denote the area-weighted number of distinct rainfall observations and the number of records in year $t$, respectively. Then, define $f_{t}=b_{t} / b_{t}$, which is an unbiased estimator of $c_{t} / \lambda_{t}$.

In general, the stations may have begun record keeping at different times. According to Eq. (4), given that everything else is equivalent, a record is less likely to be set from stations (or grids in our dataset) with longer reporting histories. To incorporate this difference, we construct cohorts of grids, depending on their start times, as follows. We group the sequences that started at time $t+1$ as cohort $h(t)$ (the earliest time series are those of cohort 0 ) (note that $\lambda_{t, h(t)}=\lambda_{t}-\lambda_{h(t)}$, where $\lambda_{0}=0$ ). Let $n_{t, h(t)}$ and $b_{t, h(t)}$ denote the area-weighted number of distinct observations and the area-weighted number of records in cohort $h(t)$, respectively, at time $t$. The logarithm of likelihood $L_{t, h(t)}(\hat{c})$ of observing $b_{t, h(t)}$ records among $n_{t, h(t)}$ observations for cohort $h(t)$ is equal to:

$$
\begin{aligned}
L_{t, h(t)}(\hat{c}) \approx & b_{t, h(t)} \ln \hat{c}_{t}+\left[n_{t, h(t)}-b_{t, h(t)}\right] \ln \hat{\lambda}_{t-1, h(t)} \\
& -n_{t, h(t)} \ln \hat{\lambda}_{t, h(t)},
\end{aligned}
$$

where $\hat{c}=\left(\hat{c}_{1}, \hat{c}_{2}, \ldots, \hat{c}_{T}\right)$. By summing the likelihoods of all of the cohorts, we obtain

$$
L(\hat{c})=\sum_{t=1}^{T} \sum_{h(t) \leq t-1} L_{t, h(t)}(\hat{c}) .
$$

By fixing $\hat{c}_{1}$ to 1 , a vector of $\hat{c}$ to maximize Eq. (5) is the number of hypothetical draws that best fit the areaweighted likelihoods of the observed records.

\section{5) RELATION BETWEEN RECORDS AND EXTREME EVENTS}

Equation (2) shows that EVT shares a theoretical foundation with our approach. Under both approaches, the observed rainfall data are assumed to be drawn from the distribution implied by multiple draws that are sampled from some underlying distribution. Under EVT, Eq. (2) is extended by taking the limit of the number of draws $\gamma(1, s)$ to infinity, which establishes the asymptotic distribution of the tails. However, when the sample size is relatively small, as seen with the Indian monsoon rainfall data, it is not obvious whether the assumed extreme rainfall is truly extreme. Therefore, errors from assuming the same asymptotic tail for the heterogeneous rainfall distributions across the region can be sizable.

Our approach instead is to identify the link between RED and the likelihood of extreme rainfall. Here, we show that the change in RED converges to the change in the likelihood of observing extreme rainfall. We normalize the high-rainfall RED (RED-H) in the base year to one and assume that the RED-H in the current year is equal to $n>0$. Assume that $Z_{1}, Z_{2}, \ldots, Z_{n}$, and $n$ draws in the current year are from a continuous and strictly increasing cumulative distribution function $F(z) \sim[\alpha, \beta]$, where $F(z)$ is the underlying rainfall distribution in the base year. Let $Z_{(k)}$ denote the $k^{\text {th }}$ smallest $Z$ such that $Z_{(1)}<Z_{(2)}<\cdots<Z_{(n-1)}<Z_{(n)}$. The probability of observing rainfall greater than $h$ (the previous record high) from a maximum of $n$ draws in the current year is given by

$$
\begin{aligned}
g_{n}(h) & =P\left(Z_{(n)}>h\right)=1-P\left(Z_{(n)}<h\right)=1-\prod_{i=1}^{n} P\left(Z_{i}<h\right) \\
& =1-F^{n}(h) .
\end{aligned}
$$

Similarly, the probability of observing rainfall greater than $h$ from a single draw in the base year is given by

$$
g_{1}(h)=1-F(h) .
$$

Then, the ratio $g_{n}(h) / g_{1}(h)$ represents the change in the likelihood of observing rainfall greater than $h$ in the current year compared with the base year. Taking the 

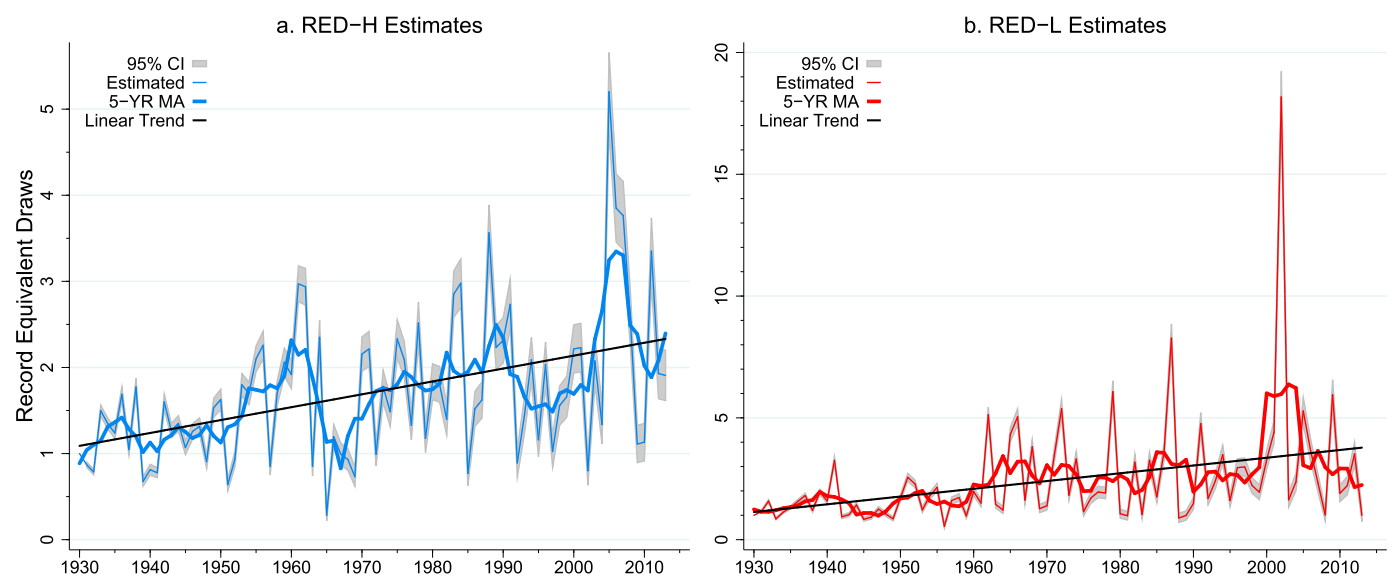

FIG. 1. (a) RED-H and (b) RED-L estimates based on monsoon rainfall during 1930-2013 throughout India. All RED estimates are normalized to 1 in 1930. The RED estimates are represented by the thin lines, and the 5-yr moving averages (MA) of the RED estimates are represented by the thick lines. The $95 \%$ confidence interval (CI) of RED estimates is based on a bootstrapping experiment where we randomly select 1000 time series of rainfall observations. The black lines represent the linear trend on RED.

limit of the ratio $g_{n}(h) / g_{1}(h)$ as $h$ approaches the upper bound of the distribution, we obtain

$$
\lim _{h \rightarrow \beta} \frac{g_{n}(h)}{g_{1}(h)}=\lim _{h \rightarrow \beta} \frac{1-F^{n}(h)}{1-F(h)}=\lim _{h \rightarrow \beta} \sum_{t=1}^{n}[F(h)]^{t-1}=n .
$$

Therefore, changes in RED-H approximate the change in the likelihood of observing an extreme high rainfall. The ratio approaches this limit monotonically as $h$ approaches extreme values because $F(\cdot)$ is strictly increasing. The same argument holds for the likelihood of observing an extreme low rainfall that is derived from changes in the low-rainfall RED (RED-L). Therefore, changes in RED measure change in the likelihood of extreme rainfall relative to the base year.

\section{6) RECORD RAINFALL DATA CONSTRUCTION}

For our empirical analysis, the record rainfall data (based on the monthly-mean rainfall) are prepared as follows. We substitute rainfall information in 1930 with the mean rainfall in the first $30 \mathrm{yr}$ (1901-30) of the period, which is used as the reference period for comparison. Therefore, RED estimates in subsequent time periods are evaluated relative to the climatological normal of 1901-30. To identify the indicators of record rainfall events for each month and grid, we select the record high and record low monthly rainfall up to that time period. The indicator for a record high (low) is set to 1 if the observed rainfall for that year is a record high (low); otherwise, the indicator is zero. Finally, the time series of record high and record low indicators are aggregated to construct the likelihood function in Eq. (5). The coefficients that maximize the likelihood functions for record high and record low data are denoted as RED-H and RED-L, respectively.

The observed rainfall records may be biased because of both the inconsistent use of measurement instruments and the location changes of the stations. However, the impact of these inconsistencies on the RED estimates is likely to be minimal for the following reasons. The original work by Pai et al. (2014) extended the method used in a previous version of this dataset (Rajeevan et al. 2006); thus, they used stations that had at least $90 \%$ of the available observations over 1901-2013. Therefore, the data are relatively consistent at each station. The main inhomogeneity in the station-level data is the change in the number of stations for creating the grid points. However, the interpolation method used in Pai et al. (2014) limits the maximum number of nearby stations to 4 and weights the observations by distance. In addition, the resulting gridded dataset has no missing observations. Therefore, the interpolation scheme minimizes the possible bias resulting from the change in the number of stations.

\section{Likelihood of extreme rainfall}

\section{a. RED-H and RED-L}

We present the RED estimates based on monsoon rainfall from 1930 to 2013 in Fig. 1. We find statistically significant linear trends in both RED estimates: RED-H and RED-L increased 2-fold and 4-fold from 1930 to 2013, respectively, based on the linear trend estimates. We interpret this result as follows: an extreme high (low) 


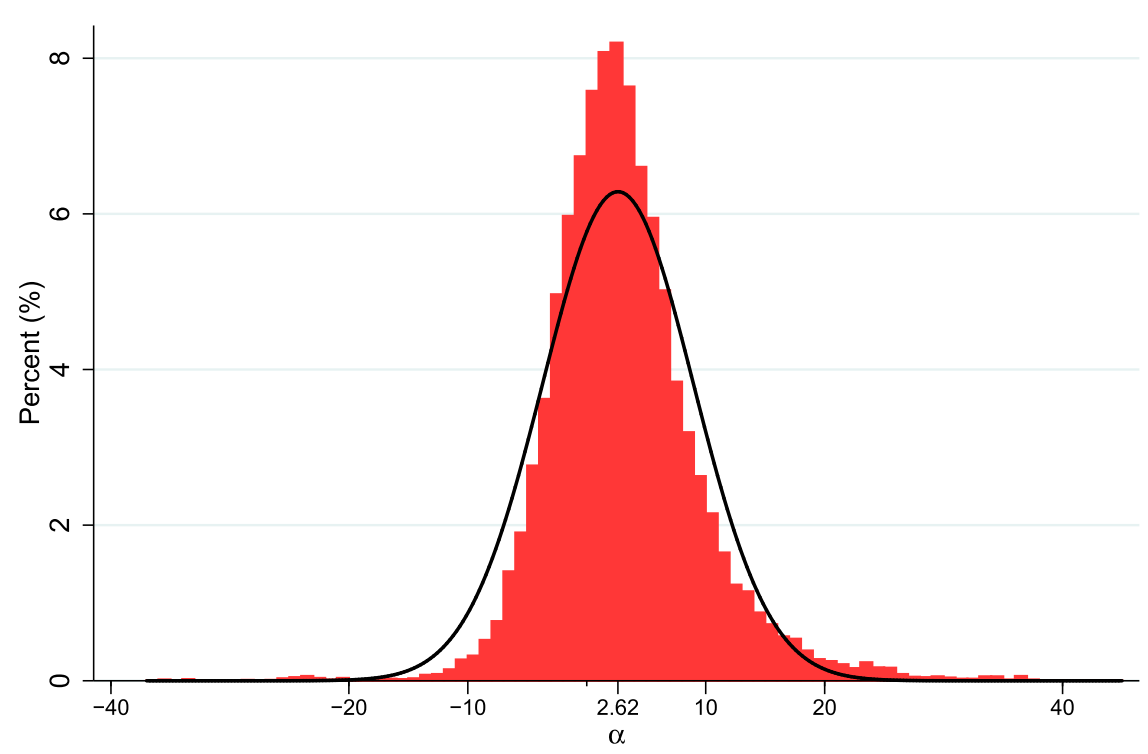

FIG. 2. The estimated distribution of the variance detector $\alpha$ based on the number of record highs and record lows. The smoothed black line represents the fit of the normal distribution. The estimated $\hat{\alpha}$ is equal to 2.62 , which indicates increasing variance. The bootstrapping method based on a random sample of 10000 observations is used to measure the statistical confidence interval for $\hat{\alpha}$. The $95 \%$ confidence interval is $(2.54,2.72)$; hence, the estimated $\hat{\alpha}$ is significantly different from zero.

rainfall event is twice (4 times) as likely to occur in 2013 as in the first $30 \mathrm{yr}$ of the past century.

A more disaggregated analysis reveals other interesting temporal patterns. In particular, during 193065, 1966-94, and 1995-2013, we observe increases in RED-H, followed by a rapid decline. The average RED-H during these time intervals increases over time from 1.4 in the first interval to 1.8 in the second interval and to 2.2 in the final interval, compared with the base time period of 1901-30 (set to 1). Thus, the overall increases are due to the dominance of the gradual increases in these distinctive time periods over the sharp declines at the end of the intervals. However, the positive linear trend of RED-L has been driven by large anomalies since 1960. For example, RED-L estimates greater than 5, which are approximately two standard deviations above the mean, only occurred after 1960 . The most dramatic increases in both RED-H and RED-L occurred in the early 2000s: a RED-H estimate of 5 in 2005 and a RED-L estimate of 18 in 2002.

To investigate further the overall changes in underlying rainfall distribution, we examine the associations between the likelihood of extreme rainfall (as measured by RED) and the mean rainfall. Note that the mean monsoon rainfall increased from 1901 to 1950 and has decreased since (Annamalai et al. 2013). Hence, the decrease in the mean rainfall since 1951 is coincident with the expansion of both tails of the rainfall distribution. Moreover, we find that the simple correlation coefficients between mean rainfall and RED-H and RED-L are 0.54 and -0.63 , respectively. The respective signs of these correlation coefficients confirm the consistency of the RED estimates: an increase in RED-H is associated with an increase in mean rainfall, and an increase in RED-L is associated with a decrease in mean rainfall. Despite the increase in RED-H, the relatively larger increases in RED-L (during 1951-2013) and relatively higher absolute value of the RED-L correlation coefficient imply a net decrease in the mean rainfall. Hence, the observed decrease in the mean rainfall since 1951 is explained by recognizing that the likelihood of extreme low rainfall has increased more than the likelihood of extreme high rainfall, rather than by suggesting that the likelihood of extreme high rainfall decreased. Consequently, our analysis of extremes highlights the fact that mean changes in rainfall can camouflage significant changes that occur at the tails of the distribution, such as the broadening of the distribution. We pursue this investigation further in the next section.

\section{b. Relationship to the variability in the distribution}

The increase in the likelihood of both extreme high and extreme low rainfall implies a broadening of the rainfall distributions across India. To test whether this broadening is significant, we compute a variance detector (Anderson and Kostinski 2010) denoted as $\alpha$ in Fig. 2. The value of $\alpha$ is defined as the area-weighted sum of the number of records (high and low) minus the same 
sum in reverse time order, and measures the changes in the variance of the underlying distributions. A significant positive value of this variance detector implies more frequent records as time progresses in comparison to the predicted rate of stationary distributions; thus, the variance increases. The advantage of this variance detector is that the result is independent of the underlying distributions; thus, it is appropriate to test whether changes in RED are associated with significant changes in the underlying variability. The results (Fig. 2) point to a statistically significant increase in the variance during 1930-2013: the estimated mean of $\alpha$ is 2.62 and is significantly different from zero.

\section{c. Comparison to the fixed threshold approach}

Our estimates of the likelihood of extreme high and extreme low rainfall are consistent with findings from previous studies using frequency counts beyond some extreme threshold value in central India. For example, Goswami et al. (2006), using rainfall observations from daily rainfall data (Rajeevan et al. 2006), also found that the frequency of very heavy rainfall (rainfall $>150 \mathrm{~mm}$ day $^{-1}$ in a grid point) in central India has been increasing since the middle of the last century.

To perform a direct comparison between the RED and the threshold methods, we use a time series of monthly rainfall observations in central India and compute the frequencies of high and low extreme rainfall based on high and low threshold values at the 5\% observed cutoffs. We find that the correlation coefficient between RED-H (RED-L) and the frequency of high (low) rainfall is 0.64 (significant at $1 \%$ ). This result confirms that our RED estimates are consistent with previous findings using fixed thresholds when the data samples are restricted to homogeneous rainfall regions during the monsoon season.

\section{Correlation analyses}

Our main objective in this section is to assess how various climate factors that are correlated with the mean monsoon rainfall might also be correlated with the likelihood of extreme rainfall. Below, we present the correlations of the RED estimates with the following seven climate factors: ENSO, IOD, IOSST, NH temperature, NAO, PDO, and Eurasian snow cover extent.

\section{a. ENSO-RED relationships}

Previous studies (Kumar et al. 1999; Wang et al. 2003) have found that monthly-mean monsoon rainfall is negatively correlated with ENSO over long historical periods. The observed negative correlation between the Indian monsoon rainfall and ENSO can be explained by changes in the zonal pattern of moisture transport by the Walker circulation (Pokhrel et al. 2012). In the warm (or positive) ENSO phase (El Niño) the ascending branch of the Walker circulation, shifts eastward in response to the anomalous warming in the central and eastern Pacific, which results in subsidence over the Indo-western $\mathrm{Pa}$ cific region. Therefore, El Niño is associated with the occurrence of below-normal monsoon rainfall. The opposite climate conditions prevail during the cold phase of ENSO (La Niña). Here, we examine whether the likelihoods of extreme high and extreme low rainfall are associated with the ENSO phases.

A simple correlation shows that the Niño-3 index is negatively (positively) correlated with the likelihood of extreme high (low) rainfall: the correlation coefficients are -0.53 and 0.48 (significant at $1 \%$ ) for RED-H and RED-L, respectively. In other words, the warm phase of ENSO is associated with a higher (lower) likelihood of extreme low (high) rainfall, and vice versa for the cold phase of ENSO.

The Niño-3 index and RED-L have been significantly correlated in recent decades, while the Niño-3 index and RED-H correlation has weakened (Fig. 3a). The negative correlation between RED-H and the Niño-3 index was significant during 1930-80, but the correlation has since weakened. In contrast, a significant positive correlation between RED-L and the Niño-3 index has been observed in the 1930s and since 1970 (an exception is in 1997, when the Niño-3 index was more than three standard deviations larger than its mean). This finding is in sharp contrast to the correlation between the mean monsoon rainfall and the Niño-3 index [i.e., the correlation has weakened since the late 1970s (Kumar et al. 1999)].

\section{b. IOD-RED relationships}

The simple correlations between the IOD index and RED-H and RED-L are 0.38 and -0.30 (significant at $1 \%)$, respectively. These correlations are consistent with the well-noted positive association between mean monsoon rainfall and the IOD (Saji et al. 1999; Pokhrel et al. 2012); the simple correlation between the IOD index and mean monsoon rainfall is 0.32 according to Saji et al. (1999). A more disaggregated analysis shows that our correlations are nonstationary over time (Fig. 3b): the IOD index is positively correlated with the likelihood of extreme high rainfall in 1975-90 and is negatively correlated with the likelihood of extreme low rainfall since the mid-1980s.

Notice that the IOD-RED-L correlation has been significant in recent decades. This observation is consistent with the recent finding (Ajayamohan and Rao 2008) of a significant negative correlation (up to -0.69) 
a. Nino3-JJAS

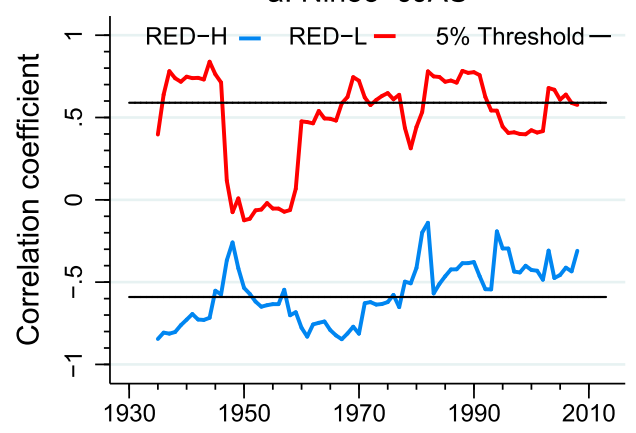

c. IOSST-MAM

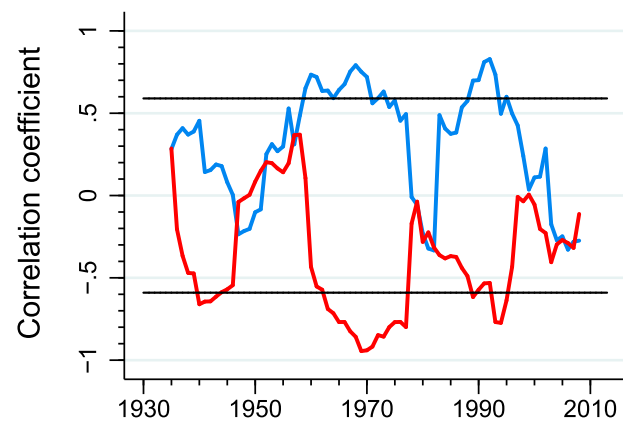

e. $\mathrm{NH}$ Temperature-MAM

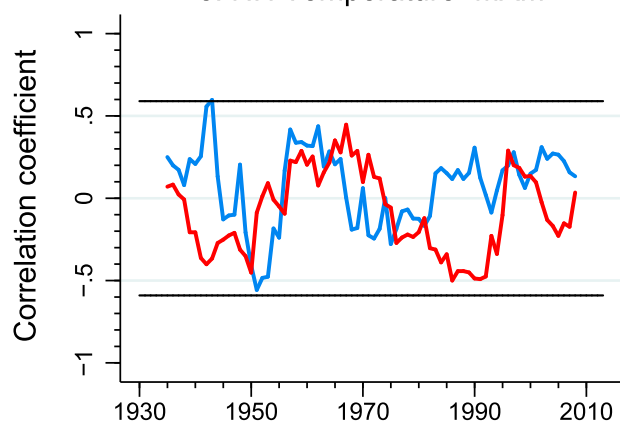

g. PDO-JJAS

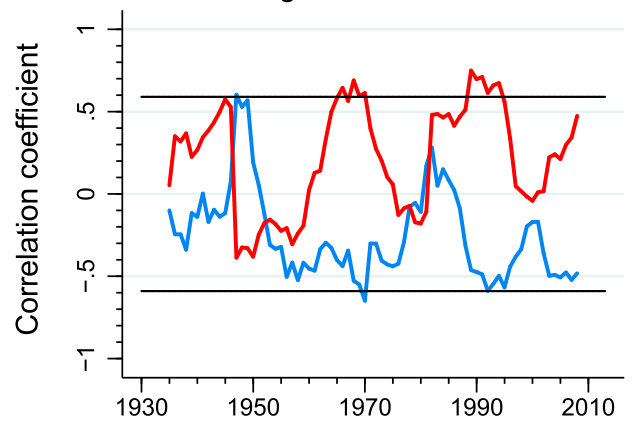

b. IOD-JJAS

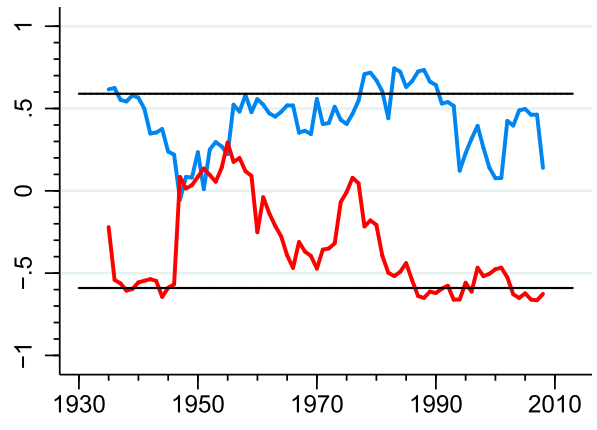

d. IOSST-JJAS

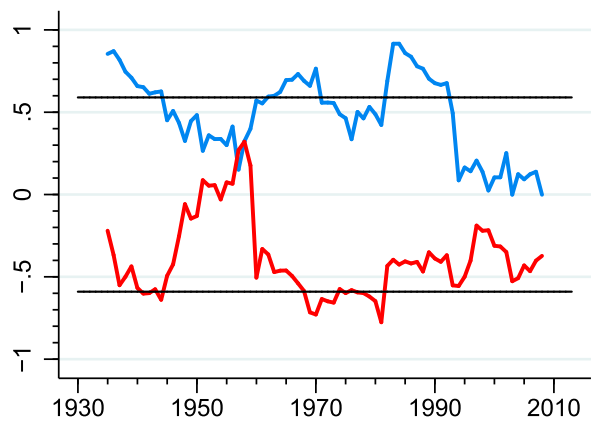

f. NH Temperature-JJAS

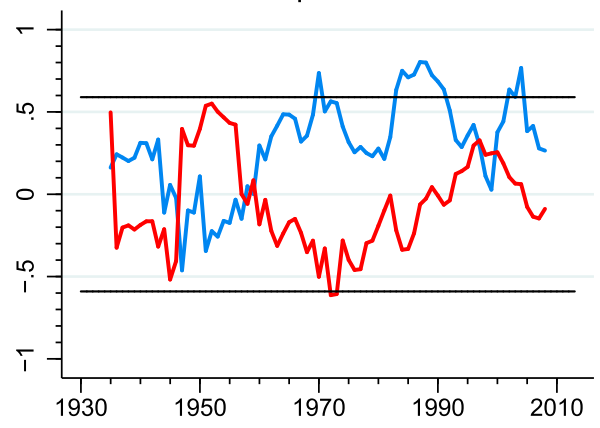

h. Snow Cover-DJF

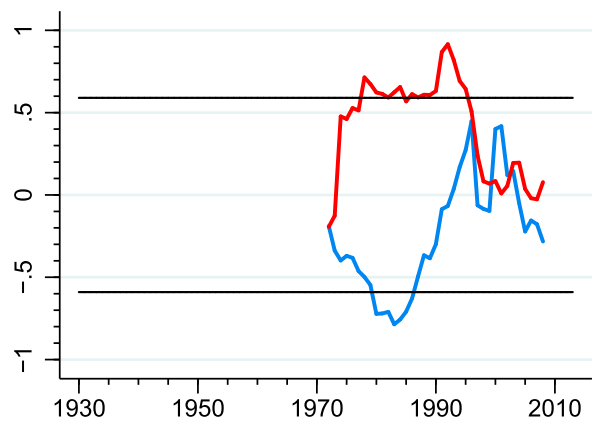

FIG. 3. The 11-yr sliding correlations between the RED estimates and six climate factors in 1930-2013. The climate factors are as follows: (a) Niño-3 in the monsoon season, (b) IOD in the monsoon season, IOSST in the (c) spring and (d) monsoon season, NH temperature in the (e) spring and (f) monsoon season, (g) PDO in the monsoon season, and (h) snow cover over western Europe in the winter. The correlations with RED-H are in blue, and the correlations with RED-L are in red. The 5\% thresholds for statistical significance are shown as black horizontal lines. The years correspond to the middle of the 11-yr period. 
between daily level heavy rainfall $\left(>100 \mathrm{~mm} \mathrm{day}^{-1}\right.$ in a grid point) and SST anomalies at the eastern edge of the IOD region $\left(10^{\circ} \mathrm{S}-0^{\circ}, 90^{\circ}-110^{\circ} \mathrm{E}\right)$. According to Ajayamohan and Rao (2008), the responsible physical mechanism is possibly the anomalously cold SST at the eastern edge of the IOD region and the associated strong easterlies, which are responsible for transporting moisture from the southeastern equatorial Indian Ocean to the Bay of Bengal. This process leads to the convergence of moisture over India.

\section{c. IOSST-RED relationships}

In this section, we examine the IOSST-RED correlations. Investigating the association between IOSST and RED is important because of the hydrological cycle: a warmer ocean evaporates more water into the atmosphere (Clark et al. 2000; Dai et al. 2001), and a portion of the water evaporated from the ocean is transported to the continent where it precipitates. Annamalai et al. (1999) show that during the monsoon season, circulations transport moisture from the surrounding Indian Ocean, particularly the Arabian Sea, to the Indian subcontinent. We test whether changes in IOSST are also associated with changes in the likelihood of extreme rainfall. To do so, we correlate the area-weighted mean sea surface temperature in the tropical Indian Ocean $\left[24^{\circ} \mathrm{S}-24^{\circ} \mathrm{N}, 40^{\circ}-120^{\circ} \mathrm{E}\right.$, as in Clark et al. (2000)] in the spring [March-May (MAM)] and in the monsoon season with RED.

We find a positive (negative) association, in general, between IOSST and RED-H (RED-L) in the spring and monsoon seasons. Upon closer examination, we find that the strength of these correlations changes over time. The correlation of the preceding spring IOSST with both RED-H and RED-L (Fig. 3c) is significant during 196075 and the early 1990s. In contrast, during the monsoon season in 1960-90, the significance of the correlations between IOSST and RED-H and RED-L alternate: RED-H is positively correlated in the 1960s and 1980s, and RED-L is negatively correlated in 1965-80 (Fig. 3d). To determine the robustness of the results, we examine these correlations within subregions of the Indian Ocean and confirm the same correlation patterns between IOSST and RED (see supplementary Fig. 1 in the supplemental material).

Since 1990, none of the correlations between IOSST and RED have been significant. According to Copsey et al. (2006), this weakening relationship is related to the increase in the sea level pressure (SLP) over the Indian Ocean, which peaked in the early 1990s. In the past half century, the Indian Ocean has undergone significant warming (Annamalai et al. 2013). In the tropics, decreasing SLP is a typical feature of the warming ocean surface. However, the Indian and Atlantic Oceans have experienced increasing SLPs over the past half century, while the central and western Pacific Oceans have experienced decreasing SLPs (Copsey et al. 2006; Annamalai et al. 2013). Therefore, the air mass shifted from the Pacific to the east and west. This increase in SLP over the Indian Ocean can override the warming of the Indian Ocean by suppressing rising air that supplies moisture. This observation implies that warming in IOSST is less efficient in generating monsoon rainfall when it is countered by increases in SLP.

\section{d. NH temperature-RED relationships}

The physical link between large-scale atmospheric warming and local precipitation, such as Indian monsoon rainfall, is difficult to establish since this relationship is jointly determined by changes in the hydrologic cycle resulting from increased warming and interaction with regional climate conditions, such as snow cover (Kumar et al. 1999; Fan et al. 2009). In this section, we examine the statistical correlation between $\mathrm{NH}$ temperature and the likelihood of extreme rainfall. Note that the $\mathrm{NH}$ temperature increased rapidly from the mid-1970s until the early 2000s; the greatest variability occurred in the midlatitudes $\left(30^{\circ}-60^{\circ} \mathrm{N}\right)$. Hence, we correlate the NH surface air temperature in the midlatitudes with RED and use both the spring and monsoon season temperatures, which are correlated with mean monsoon rainfall (Kumar et al. 1999).

We find that the $\mathrm{NH}$ temperatures in the spring (MAM) and RED are not significantly correlated in the past century (Fig. 3e). In the concurrent monsoon season, the $\mathrm{NH}$ temperature and RED-H correlation is significantly positive around the late 1960s, the 1980s, and the early 2000s, while the correlation with RED-L is insignificant throughout the entire time period (Fig. 3f).

Because both $\mathrm{NH}$ temperature and monsoon rainfall are associated with ENSO (Fan et al. 2009), the correlation between the $\mathrm{NH}$ temperature and RED is likely to be complicated by the ENSO-monsoon relationship. To measure the NH temperature-RED correlation net of ENSO variability, we low-pass filtered both time series-RED and NH temperature-via Baxter and King (1999) to remove signals at frequencies shorter than $7 \mathrm{yr}$, since ENSO typically occur on a time scale of 3-7 yr. We then computed simple correlations with the filtered time series over the entire sample period (rather than timevarying correlations, as in Fig. 3). The results show that the simple correlation between the filtered $\mathrm{NH}$ temperature in the monsoon season and filtered RED-L is significant, while the other correlations remain unaffected by filtering. Specifically, the correlations with RED-L are 0.18 (insignificant) and -0.24 (significant at 
$1 \%)$ before and after applying the filter, respectively. This finding suggests that the negative correlation between $\mathrm{NH}$ temperature in the monsoon season and the likelihood of extreme low rainfall is obscured by the ENSO effect.

Since the NH temperature in the spring season is not strongly correlated with the likelihood of extreme rainfall, it is interesting to examine whether the underlying atmospheric variability in the premonsoon season might be associated with the likelihood of extreme rainfall. One source of variability could arise from the NAO. The NAO is significantly associated with the temperature and precipitation of the winter season in regions from eastern North America to western and central Europe (van Loon and Rogers 1978; Robock et al. 2003). A physical mechanism for this association is that the positive phase of the NAO induces stronger westerlies that advect warmer ocean air over the land regions; the result is warm surface air temperature anomalies over Europe and eastern North America, and vice versa for the negative phase of the NAO.

In this study, we test whether the strong NAO's association with atmospheric variability in the winter season might be extended to a significant correlation with the likelihood of extreme rainfall in the monsoon season. We find that the NAO-RED correlations are insignificant for both RED-H and RED-L (see supplementary Fig. 2 in the supplemental material). This result is consistent with the observation by Hori and Yasunari (2003), who found that atmospheric variability caused by the NAO in the winter does not last long enough to correlate with extreme monsoon rainfall, and the finding from climate models that the NAO-monsoon association is random (Robock et al. 2003). This result parallels with our observation of the weak correlation between $\mathrm{NH}$ temperature in the spring and RED estimates.

\section{e. PDO-RED relationships}

The PDO, which cycles every $20-30 \mathrm{yr}$, influences the variability in the tropical Pacific Ocean and thus determines the interannual variability in monsoon rainfall in Asia. Previous studies (Chan and Zhou 2005; Krishnamurthy and Krishnamurthy 2014) show a significant negative correlation exists between the PDO and mean monsoon rainfall.

Although the PDO is most influential in the North Pacific, it produces circulation patterns that are similar to ENSO, both temporally and spatially. When the PDO is in the positive phase, the SST is anomalously cool in the interior North Pacific and warm along the Pacific coast. Similarly, in the warm phase of ENSO, abnormally warm SSTs occur from the date line (i.e. $180^{\circ}$ longitude) to the South American coast. The climate conditions reverse in the cold phases of the PDO and ENSO. Because of the similarity between the PDO and ENSO, we examine whether the PDO has any significant correlation with RED and whether the correlation is similar to the ENSO-RED association.

We find that the PDO is generally negatively (positively) correlated with RED-H (RED-L), which is consistent with the patterns of the ENSO-RED correlations (in Fig. 3a). A more detailed analysis shows that the statistical significance of these correlations changes over time (Fig. 3g); the likelihood of extreme low rainfall is positively associated with the PDO in 1965-70 and in 1989-95. The likelihood of extreme high rainfall is negatively associated with PDO for short time periods in the late 1960s and early 1990s.

Interestingly, the troughs of the PDO-RED-L correlation occurred at approximately the time when the PDO changed phases. Specifically, the PDO switched phases in 1925, 1947, 1977 (Mantua et al. 1997), and 1998. The troughs of the PDO and RED-L correlation occurred in 1947, 1977, and 1999. Therefore, the PDO and RED-L correlation has the same periodicity as the PDO.

Notably, the PDO and ENSO may influence each other (Newman et al. 2003). In particular, the warm phases of the PDO and ENSO can complement each other to induce further subsidence over the Indian subcontinent, and vice versa for the cool phase of the PDO and ENSO. To test whether the co-occurrence of PDO and ENSO phases enhances the likelihood of extreme rainfall, we divide the entire sample period of 1930-2013 into three categories: warm, cold, and neutral phases. The warm and cold phases are years when both Niño-3 and PDO indices are in the upper and lower quartiles of all observations in 1930-2013, respectively. The neutral phases compose the rest of the sample. The number of years with warm, cold, and neutral phases are 6,9 , and 69, respectively. We compare the mean RED-H and RED-L for each of the three categories. We find that the mean likelihood of extreme high rainfall is highest in cold phases, followed by neutral and warm phases; the opposite is true for RED-L [i.e., the mean likelihood of extreme low rainfall is highest in warm phases, followed by neutral and warm phases (see supplementary Table 1 in the supplemental material)]. This result is robust for different thresholds of warm and cold phases. Therefore, the likelihood of an extreme rainfall event, either high or low, may be enhanced when ENSO and the PDO are in the same phase. This result is consistent with the relationship among the triad-PDO, ENSO, and monsoon rainfall-in South China (Chan and Zhou 2005; Zhou et al. 2006) where summer monsoon rainfall over South China are more likely to be below (above) normal when both ENSO and the PDO 
are in the warm (cold) phase, while the rainfall show no particular deviation from normal when the two indices are in the opposite phase.

\section{f. Eurasian snow cover-RED relationship}

Previous studies show that snow cover in the preceding winter and spring over Eurasia is, in general, negatively correlated with the mean monsoon rainfall (Robock et al. 2003; Peings and Douville 2010). The negative correlation is confirmed by a number of numerical simulations using general circulation models (Barnett et al. 1989). However, large variability occurs in the strength of the correlation across different regions of Eurasia because of differences in local climate conditions (Fasullo 2004; Peings and Douville 2010). Therefore we choose the area-weighed mean SCE in the winter [December-February (DJF)] in western Europe $\left(45^{\circ}-55^{\circ} \mathrm{N}, 0^{\circ}-10^{\circ} \mathrm{E}\right)$, where the correlation between SCE and the mean monsoon rainfall is strongest within Eurasia (Robock et al. 2003; Fasullo 2004).

We find (Fig. 3h) that a significant positive correlation existed between SCE and RED-L in 1980-95, and a significant negative correlation existed between SCE and RED-H in the mid-1980s. We also confirm that these RED correlations with snow cover are the most significant in western Europe compared with other parts of Eurasia (see supplementary Fig. 3 in the supplemental material). However, no significant correlations have been observed since the mid-1990s.

The lack of significant correlations between RED and SCE in recent decades is partly related to the ENSOmonsoon rainfall association. Previous studies (Fasullo 2004; Peings and Douville 2010) show that the relationship between Eurasian snow cover and monsoon rainfall is overwhelmed (or disrupted) during strong ENSO years. Specifically, observations indicate that the regions with a significant snow cover and monsoon correlation broadly coincide with the regions with significant correlations between snow cover and ENSO. Thus, the observed correlation (or lack of correlation) between SCE and monsoon rainfall could be related to the SCE-ENSO association.

To test whether SCE-RED correlations become stronger after removing the influence of ENSO variability, we apply a low-pass filter to both the time series of SCE and RED to eliminate the influence of ENSO and thus retain only the variations of periods greater than $7 \mathrm{yr}$ (see section $4 \mathrm{~d}$ for more details on filtering process). We then estimate simple correlations between the filtered RED and filtered SCE. The results show that the SCE and RED-H correlations become stronger when the two time series are filtered. The correlation with the filtered time series is -0.33 (significant at $1 \%$ ), while it is only -0.18 (insignificant) with the unfiltered series. By contrast, the SCE and RED-L correlation remains unchanged after filtering. Therefore, a stronger SCE and RED-H correlation may exist, but it is obscured by the presence of ENSO.

\section{Summary and discussion}

In this study, we develop a new metric to measure the likelihood of extreme events without needing the specifics of the underlying distributions. The method is particularly useful for examining the overall changes in extreme rainfall patterns in India, where rainfall distributions are heterogeneous. Our approach builds upon several findings from previous studies that rely on defined thresholds to identify extreme rainfall events and that are restricted to regions with homogeneous rainfall distributions, such as central India. Using our metric, we find a substantial increase in both the likelihood of extreme high and extreme low rainfall over the past century; the extremes reached a peak in the early 2000s. We confirm that the increase in the likelihood of extreme rainfall is associated with the increase in the variance of the rainfall distributions.

Our correlation analyses reveal that in the recent decades, the likelihood of extreme low rainfall is positively correlated with ENSO and negatively correlated with IOD. The weak correlations in recent decades between climate factors, such as IOSST and Eurasian snow cover, and the likelihood of extreme rainfall are associated with countervailing forces from increased sea level pressure over the Indian Ocean and the opposite phase of ENSO, as argued in several other studies. Moreover, the temporal patterns of the likelihood of extreme rainfall exhibit multidecadal variability: RED-H exhibits approximately three-decade-long periodicity in 1930-65, 1966-94, and 1995-2013; and RED-L is generally higher in the period 1960-90 than in the previous three decades. This implies that the strong correlations of RED in the late 1960s and around 1990 with Niño-3, IOSST, and the PDO mostly coincide with periods of transition between extreme wet and extreme dry decades of monsoon rainfall. Therefore, further investigation of the physical base for decadal behavior and phase changes of extreme rainfall will be useful in predicting future extreme monsoon rainfall.

Although the association between ENSO and the likelihood of extreme rainfall has been significant in recent decades, the likelihood of extreme rainfall in the future is uncertain, particularly because of our lack of understanding of the future changes in ENSO intensity. Some studies (Turner et al. 2007) have suggested a possible increase in the ENSO amplitude in the future, 
while other studies (van Oldenborgh et al. 2005) have suggested no significant changes. Therefore, further research is needed to predict the likelihood of extreme monsoon rainfall in the future.

Acknowledgments. We gratefully acknowledge financial support from the Melon Climate Research Fund 2013 from the Barnard College, Columbia University. The first author would like to thank Barnard College, Columbia University for providing invaluable support to conduct research on climate change while he visited the institution for the summers of 2012 and 2013. We also thank Mark Cane of the Lamont-Doherty Earth Observatory, Columbia University for encouragement and generous feedback on earlier versions of the paper.

\section{REFERENCES}

Ajayamohan, R. S., and S. A. Rao, 2008: Indian Ocean Dipole modulates the number of extreme rainfall events over India in a warming environment. J. Meteor. Soc. Japan, 86, 245-252, doi:10.2151/jmsj.86.245.

Anderson, A., and A. B. Kostinski, 2010: Reversible record breaking and variability: Temperature distributions across the globe. J. Appl. Meteor. Climatol., 49, 1681-1691, doi:10.1175/ 2010JAMC2407.1.

Annamalai, H., J. M. Slingo, K. R. Sperber, and K. Hodges, 1999: The mean evolution and variability of the Asian summer monsoon: Comparison of ECMWF and NCEP-NCAR reanalyses. Mon. Wea. Rev., 127, 1157-1186, doi:10.1175/ 1520-0493(1999)127<1157:TMEAVO>2.0.CO;2.

_ J. Jafner, K. P. Sooraj, and P. Pillai, 2013: Global warming shifts the monsoon circulation, drying South Asia. J. Climate, 26, 2701-2718, doi:10.1175/JCLI-D-12-00208.1.

Barnett, T. P., L. Dümenil, U. Schlese, E. Roeckner, and M. Latif, 1989: The effect of Eurasian snow cover on regional and global climate variations. J. Atmos. Sci., 46, 661-686, doi:10.1175/ 1520-0469(1989)046<0661:TEOESC $>2.0$. CO;2.

Baxter, M., and R. G. King, 1999: Measuring business cycles: Approximate band-pass filters for economic time series. Rev. Econ. Stat., 81, 575-593, doi:10.1162/003465399558454.

Benestad, R. E., 2003: How often can we expect a record event? Climate Res., 25, 3-13, doi:10.3354/cr025003.

Chan, J. C. L., and W. Zhou, 2005: PDO, ENSO and the early summer monsoon rainfall over South China. Geophys. Res. Lett., 32, L08810, doi:10.1029/2004GL022015.

Chandler, K. N., 1952: The distribution and frequency of record values. J. Roy. Stat. Soc., 14B, 220-228.

Clark, C. O., J. E. Cole, and P. J. Webster, 2000: Indian Ocean SST and Indian summer rainfall: Predictive relationships and their decadal variability. J. Climate, 13, 2503-2519, doi:10.1175/ 1520-0442(2000)013<2503:IOSAIS >2.0.CO;2.

Copsey, D., R. Sutton, and J. R. Knight, 2006: Recent trends in sea level pressure in the Indian Ocean region. Geophys. Res. Lett., 33, L19712, doi:10.1029/2006GL027175.

Dai, A., T. M. Wigley, B. A. Boville, J. T. Kiehl, and L. E. Buja, 2001: Climates of the 20th and 21st centuries simulated by the NCAR Climate System Model. J. Climate, 14, 485-519, doi:10.1175/1520-0442(2001)014<0485:COTTAT>2.0.CO;2.
Fan, F., M. E. Mann, and C. M. Ammann, 2009: Understanding changes in the Asian summer monsoon over the past millennium: Insights from a long-term coupled model simulation. J. Climate, 22, 1736-1748, doi:10.1175/2008JCLI2336.1.

Fasullo, J., 2004: A stratified diagnosis of the Indian monsoonEurasian snow cover relationship. J. Climate, 17, 1110-1122, doi:10.1175/1520-0442(2004)017<1110:ASDOTI>2.0.CO;2.

Gadgil, S., and K. R. Kurma, 2006: The Asian monsoon-Agriculture and economy. The Asian Monsoon, B. Wang, Ed., Springer-Verlag, 651-683.

Ghosh, S., V. Luniya, and A. Gupta, 2009: Trend analysis of Indian summer monsoon rainfall at different spatial scales. Atmos. Sci. Lett., 10, 285-290, doi:10.1002/asl.235.

_ D. Das, S.-C. Kao, and A. R. Ganguly, 2012: Lack of uniform trends but increasing spatial variability in observed Indian rainfall extremes. Nat. Climate Change, 2, 86-91, doi:10.1038/ nclimate1327.

Goswami, B. N., V. Venugopal, D. Sengupta, M. S. Madhusoodanan, and P. K. Xavier, 2006: Increasing trend of extreme rain events over India in a warming environment. Science, 314, 1442-1445, doi:10.1126/science.1132027.

Guhathakurta, P., O. P. Sreejith, and P. A. Menon, 2011: Impact of climate change on extreme rainfall events and flood risk in India. J. Earth Syst. Sci., 120, 359-373, doi:10.1007/s12040-011-0082-5.

Hansen, J., R. Ruedy, M. Sato, and K. Lo, 2010: Global surface temperature change. Rev. Geophys., 48, RG4004, doi:10.1029/ 2010RG000345.

Hori, M. E., and T. Yasunari, 2003: NAO impact towards the springtime snow disappearance in the western Eurasian continent. Geophys. Res. Lett., 30, 1977, doi:10.1029/2003GL018103.

Hurrell, J. W., 1995: Decadal trends in the North Atlantic Oscillation: Regional temperatures and precipitation. Science, 269, 676-679, doi:10.1126/science.269.5224.676.

Kennedy, J. J., N. A. Rayner, R. O. Smith, D. E. Parker, and M. Saunby, 2011a: Reassessing biases and other uncertainties in sea surface temperature observations measured in situ since 1850: 1. Measurement and sampling uncertainties. J. Geophys. Res., 116, D14103, doi:10.1029/2010JD015218.

$\longrightarrow,-,-\longrightarrow$, and,$- 2011 \mathrm{~b}$ : Reassessing biases and other uncertainties in sea surface temperature observations measured in situ since 1850: 2. Biases and homogenization. J. Geophys. Res., 116, D14104, doi:10.1029/2010JD015220.

Krishnamurthy, C. K. B., U. Lall, and H.-H. Kwon, 2009: Changing frequency and intensity of rainfall extremes over India from 1951 to 2003. J. Climate, 22, 4737-4746, doi:10.1175/2009JCLI2896.1.

Krishnamurthy, L., and V. Krishnamurthy, 2014: Influence of PDO on South Asian summer monsoon and monsoonENSO relation. Climate Dyn., 42, 2397-2410, doi:10.1007/ s00382-013-1856-z.

Kumar, K. K., B. Rajagopalan, and M. A. Cane, 1999: On the weakening relationship between the Indian monsoon and ENSO. Science, 284, 2156-2159, doi:10.1126/science.284.5423.2156.

Kumar, K. N., M. Rajeevan, D. S. Pai, A. K. Srivastava, and B. Preethi, 2013: On the observed variability of monsoon droughts over India. Wea. Climate Extremes, 1, 4250, doi:10.1016/j.wace.2013.07.006.

Mantua, N. J., S. R. Hare, Y. Zhang, J. M. Wallace, and R. C. Francis, 1997: A Pacific interdecadal climate oscillation with impacts on salmon production. Bull. Amer. Meteor. Soc., 78, 1069-1079, doi:10.1175/1520-0477(1997)078<1069: APICOW $>2.0 . \mathrm{CO} ; 2$.

Martinez-Casasnovas, J. A., M. C. Ramos, and M. Ribes-Dasi, 2002: Soil erosion caused by extreme rainfall events: Mapping 
and quantification in agricultural plots from very detailed digital elevation models. Geoderma, 105, 125-140, doi:10.1016/ S0016-7061(01)00096-9.

Meehl, G. A., C. Tebaldi, G. Walton, D. Easterling, and L. McDaniel, 2009: Relative increase of record high maximum temperatures compared to record low minimum temperatures in the U.S. Geophys. Res. Lett., 36, L23701, doi:10.1029/2009GL040736.

Min, S.-K., X. Zhang, F. W. Zwiers, and G. C. Hegerl, 2011: Human contribution to more-intense precipitation extremes. Nature, 470, 378-381, doi:10.1038/nature09763.

Newman, M., G. P. Compo, and M. A. Alexander, 2003: ENSOforced variability of the Pacific decadal oscillation. J. Climate, 16, 3853-3857, doi:10.1175/1520-0442(2003)016<3853: EVOTPD $>2.0 . \mathrm{CO} ; 2$.

Pai, D. S., L. Sridhar, M. Rajeevan, O. P. Sreejith, N. S. Satbhai, and B. Mukhopadhyay, 2014: Development of a new high spatial resolution $\left(0.25^{\circ} \times 0.25^{\circ}\right)$ long period (1901-2010) daily gridded rainfall data set over India and its comparison with existing data sets over the region. Mausam, 65, 1-18.

Peings, Y., and H. Douville, 2010: Influence of the Eurasian snow cover on the Indian summer monsoon variability in observed climatologies and CMIP3 simulations. Climate Dyn., 34, $643-$ 660, doi:10.1007/s00382-009-0565-0.

Pokhrel, S., H. S. Chaudhari, S. K. Saha, A. Dhakate, R. K. Yadav, K. Salunke, S. Mahapatra, and S. A. Rao, 2012: ENSO, IOD and Indian summer monsoon in NCEP climate forecast system. Climate Dyn., 39, 2143-2165, doi:10.1007/s00382-012-1349-5.

Rajeevan, M., J. Bhate, J. D. Kale, and B. Lal, 2006: High resolution daily gridded rainfall data for the Indian region: Analysis of break and active monsoon spells. Curr. Sci., 91, 296-306. [Available online at http://www.iisc.ernet.in/currsci/aug102006/ 296.pdf.]

,-- , and A. K. Jaswal, 2008: Analysis of variability and trends of extreme rainfall events over India using 104 years of gridded daily rainfall data. Geophys. Res. Lett., 35, L18707, doi:10.1029/2008GL035143.

Rayner, N. A., D. E. Parker, E. B. Horton, C. K. Folland, L. V. Alexander, D. P. Rowell, E. C. Kent, and A. Kaplan, 2003:
Global analyses of sea surface temperature, sea ice, and night marine air temperature since the late nineteenth century. J. Geophys. Res., 108, 4407, doi:10.1029/2002JD002670.

Rényi, A., 1962: Theorie des éléments saillants d'une suite d'observations. Colloquium on Combinatorial Methods in Probability Theory, Aarhus, Denmark, Matematisk Institut, 104-117.

Robock, A., M. Mu, K. Vinnikov, and D. Robinson, 2003: Land surface conditions over Eurasia and Indian summer monsoon rainfall. J. Geophys. Res., 108, 4131, doi:10.1029/2002JD002286.

Saji, N. H., B. N. Goswami, P. N. Vinayachandran, and T. Yamagata, 1999: A dipole mode in the tropical Indian Ocean. Nature, 401, 360-363.

Turner, A. G., P. M. Inness, and J. M. Slingo, 2007: The effect of doubled $\mathrm{CO}_{2}$ and model basic state biases on the monsoonENSO system. I: Mean response and interannual variability. Quart. J. Roy. Meteor. Soc., 133,1143-1157, doi:10.1002/qj.82.

van Loon, H., and J. C. Rogers, 1978: The seesaw in winter temperatures between Greenland and northern Europe. Part I: General description. Mon. Wea. Rev., 106, 296-310, doi:10.1175/ 1520-0493(1978)106<0296:TSIWTB > 2.0.CO;2.

van Oldenborgh, G. J., S. Y. Pillip, and M. Collins, 2005: El Niño in a changing climate: A multi-model study. Ocean $S c i ., 1,81-95$, doi:10.5194/os-1-81-2005.

Wang, B., R. Wu, and T. Li, 2003: Atmosphere-warm ocean interaction and its impacts on Asian-Australian monsoon variation. J. Climate, 16, 1195-1211, doi:10.1175/1520-0442(2003)16<1195: AOIAII $>2.0 . \mathrm{CO} ; 2$

Yang, M. C. K., 1975: On the distribution of the inter-record times in an increasing population. J. Appl. Probab., 12, 148-154, doi: $10.2307 / 3212417$

Zhou, W., C. Li, and J. C. L. Chan, 2006: The interdecadal variations of the summer monsoon rainfall over South China. Meteor. Atmos. Phys., 93, 165-175, doi:10.1007/s00703-006-0184-9.

Zwiers, F. W., and V. V. Kharin, 1998: Changes in the extremes of the climate simulated by CCC GCM2 under $\mathrm{CO}_{2}$ doubling. J. Climate, 11, 2200-2222, doi:10.1175/1520-0442(1998)011<2200: CITEOT $>2.0 . \mathrm{CO} ; 2$ 